\title{
Partial second toe pulp free flaps in early childhood
}

\author{
Min Ki Hong, Dong Chul Lee, Min Suk Choi, Sung Hoon Koh, Jin Soo Kim, Si Young Roh, \\ Kyung Jin Lee \\ Department of Plastic and Reconstructive Surgery, Gwangmyeong Sungae Hospital, Gwangmyeong, Korea
}

Background The introduction of the partial second toe pulp free flap has enabled superior aesthetic and functional results for fingertip reconstruction in adults. Children undergoing fingertip amputation for various reasons have limited options for reconstruction. Conventional treatment could shorten the finger, leading to poor cosmesis and function. We report 18 years of our experiences with fingertip reconstruction using partial second toe pulp free flaps in patients in early childhood.

Methods Medical charts of children who had undergone fingertip reconstruction using partial second toe pulp free flaps from 2001 to 2018 were retrospectively reviewed. The surgical procedures were identical to those for adults, except for the usage of 11-0 nylon sutures. Patients' demographic data, vessel size, flap dimensions, length of the distal phalanx, and functional outcomes over the course of long-term follow-up were documented. The statistical analysis was performed with the Student t-test, the Mann-Whitney U test, and Pearson correlation analysis.

Results Eighteen toe pulp flaps in 17 patients (mean age, 3.0 years) were identified. All the flaps survived without any major complications. In long-term follow-up, the flap-covered distal phalanges showed growth in line with regular development. There was no donor-site morbidity, and all children adapted to daily life without any problems. In two-point discrimination tests, the fingertip sensation recovered to almost the same level as that in the contralateral finger.

Conclusions Partial second toe pulp free flaps are an excellent option for fingertip reconstruction in young children, as well as in adults.

Keywords Free tissue transfer flaps / Childhood / Fingertip / Reconstructive surgical procedure
Correspondence: Dong Chul Lee Department of Plastic and Reconstructive Surgery, Gwangmyeong Sungae Hospital, 36 Digital-ro, Gwangmyeong 14241, Korea

Tel: +82-2-2680-7637

Fax: +82-2-2615-7218

E-mail: ophand@gmail.com

This article was presented at the 77th Congress of the Korean Society of Plastic and Reconstructive Surgeons, on November 8-10, 2019, in Seoul, Korea.

Received: June 24, 2020 • Revised: September 23, 2020 • Accepted: September 29, 2020

pISSN: 2234-6163 • elSSN: 2234-6171 • https://doi.org/10.5999/aps.2020.01137• Arch Plast Surg 2020;47:590-596

\section{INTRODUCTION}

The activities and environment of young children are carefully controlled by their caregivers; however, sometimes, inattention can result in injuries to children. The hands are a common injury site, and fingertip injuries make up approximately two-thirds of hand injuries in the pediatric population $[1,2]$. Fingertip amputation is especially challenging due to the small size of chil- dren's digits and the mechanism of these injuries, which are most frequently caused by the finger being crushed in a door joint [3]. In some cases, patients arrive at the clinic without the amputated stump. Replantation occasionally fails and results in exposure of the distal phalanx. Among the limited treatment modalities to cover these defects in children, secondary intention or revision amputation is a commonly used reconstruction method because of young patients' excellent tissue regeneration 
ability [4]. Healing by secondary intention is an easier choice of treatment, but the authors have observed that some children who had been treated with secondary intention exhibited deformed nails and shortened fingertips, which caused them distress in their school life [5].

Microsurgical treatment for early childhood fingertip injuries is a promising alternative approach, but may be troublesome in terms of the surgical technique and postoperative care. Nonetheless, we treated these defects using partial second toe pulp free flaps and evaluated the benefits and limitations of this treatment option. The aim of this study was to evaluate the efficacy of partial second toe pulp free flaps as a reconstructive method for fingertip injuries in early childhood.

\section{METHODS}

\section{Study design}

This study was a single-center retrospective chart review. The protocol was approved by our hospital's Ethics Review Board (IRB No. KIRB-2019-N-010), and informed consent for undergoing the procedures was obtained from all patients and parents.

\section{Patients}

From 2001 to 2018, among patients who visited our hospital with fingertip amputation, we identified patients who were in early childhood (defined as being under 6 years old, an age that shows rapid growth in height and weight and establishment of handedness) [6].

Patients in whom replantation failed or for whom the amputated part could not be found, resulting in distal phalanx exposure, were included in the study. Treatment options for covering the exposed distal phalanx were discussed thoroughly with the parents, and treatment was limited to patients with a strong desire to maintain the finger length and those who presented a relatively low risk for general anesthesia. Their charts were reviewed retrospectively, and they were contacted via telephone and given a standardized questionnaire.

\section{Inclusion and exclusion criteria}

Patients who visited our hospital from 2001 to 2018, were under 6 years of age, and underwent treatment with a partial second toe pulp free flap for coverage of a fingertip defect were included, while those with less than 6 months of follow-up data after the operation were excluded.

We collected information on the patients' demographics, follow-up period, operation time, diameter of the vessel, dimensions of the flap, and length of the injured and uninjured distal phalanges at the time of injury through a review of previous medical records and radiographs. Details on the dimensions of the flap and the length of the injured and uninjured distal phalanges at the time of the last follow-up were also collected. Gait and daily activity (as markers of donor complications), sensory recovery (evaluated through the two-point discrimination test), and satisfaction of patients or parents with the functioning of the digit in daily life and the appearance of the digit were checked and documented. In situations where the patient could not visit the clinic for follow-up, satisfaction and flap status were checked through telephone surveys.

\section{Surgical technique}

All procedures were performed under general anesthesia. After the recipient finger was adequately debrided, the shape and dimensions of the defect were measured. At the donor site, a teardrop-shaped flap was designed on the medial side of the toe. The design did not proceed beyond the midline of the toe to ensure that primary closure of the donor site would be possible [7].

The incision was made along the designed line with care taken to avoid damaging the subcutaneous vein. As the vein is located just beneath the deep dermis, it was necessary for the surgeon to lightly squeeze the foot to visualize the subcutaneous vein. The subcutaneous vein was present in all cases. It can be harvested as needed, as each vein is compatible with the subcutaneous vein in the finger. After identification of the subcutaneous vein, the dissection progressed towards the medial side of the neurovascular bundle. From the distal tip, the flap was detached from the distal phalanx with sharp dissection through the pre-tendinous layer. During dissection, the neurovascular bundle carefully saved and then the pedicle was divided at the level of the proximal phalanx near the metatarsophalangeal joint with ligation of the distal end of the digital artery [8]. All procedures were conducted under loupe magnification.

The flap included the medial plantar digital artery, digital nerve, and subcutaneous vein. We anastomosed the vessels to the volar subcutaneous veins and digital artery of the recipient finger. The donor-toe incision was then closed. For adult patients, 9-0 or 10-0 nylon was usually used, but for children, 11-0 nylon was used for microanastomosis due to the small diameter of the vessels (Fig. 1).

Postoperative care was similar to that for usual replantation cases, with heparin injections every 4 hours for 1 week, prostaglandin $\mathrm{E} 1$ daily for the first 5 days, and intravenous antibiotics for 1 week with the dose adjusted according to the child's weight. Absolute bed rest was recommended for 1 week.

\section{Statistical analysis}

The correlation between the dimensions of the grown flap and 


\section{Fig. 1. Partial second toe free flap design and elevation}

(A) Flap design on the medial side of the second toe. (B) Flap elevation and harvesting along the pre-tendinous layer with neurovascular bundle.

(C) Donor site was closed primarily.
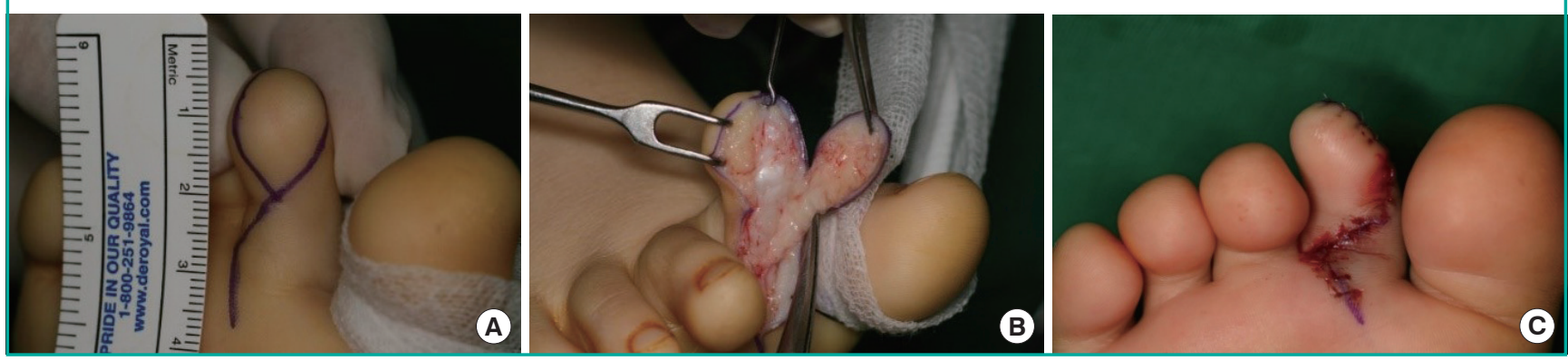

Table 1. Data of pediatric patients who underwent partial second toe free pulp transfer documented by chart review $(n=18)$

\begin{tabular}{|lc|}
\hline Factor & Mean (range) \\
\hline Age $(\mathrm{yr})$ & $3.0(1-5)$ \\
Sex, male/female & $10 / 7$ \\
Hand, right/left & $12 / 6$ \\
Finger, index/middle/ring/little & $4 / 8 / 5 / 1$ \\
Diameter of artery (mm) & $0.5(0.4-0.7)$ \\
Diameter of vein (mm) & $0.3(0.3-0.5)$ \\
Operation time (min) & $142(105-240)$ \\
Follow-up period (mon) & $66.4(6-213)$ \\
Flap size (cm²) & $1.72(0.96-2.34)$ \\
\hline
\end{tabular}

distal phalanx length was analyzed. The growth rate of the distal phalanx and sensory recovery (evaluated through the two-point discrimination test) were compared between the injured finger and contralateral uninjured finger. Statistical analyses were performed with IBM SPSS Statistics for Windows, version 25.0 (IBM Corp., Armonk, NY, USA) using the Student t-test, the Mann-Whitney U test, and Pearson nonparametric coefficient correlation analysis. A P-value of less than 0.05 was considered to indicate statistical significance.

\section{RESULTS}

From 2001 to 2018, 133 patients below 6 years of age visited our emergency center with fingertip amputation. Replantation was attempted in 112 cases, and among them, 14 cases needed free flap coverage due to distal phalanx exposure after replantation. In four of the 21 patients who visited without the amputated stump or with a severely crushed stump, the fingertip was immediately covered with a partial second toe pulp free flap.

Eighteen digits from 17 patients who fulfilled all the criteria were identified, with one patient undergoing two operations on the right index and middle fingers (a 2-year-old boy). All the patients were either toddlers $(1-2$ years, $n=7)$ or preschoolers
Table 2. Long-term follow-up data of traceable patients $(n=15)$

\begin{tabular}{|ll|}
\hline Characteristics & Mean (range) \\
\hline Growth rate (\%) & \\
$\quad$ Flap dimension & $68.9(19.7-182.0)$ \\
$\quad$ Distal phalanx of injured finger & $43.2(0-93.1)$ \\
$\quad$ Distal phalanx of uninjured finger & $47.6(0-95.4)$ \\
Static two-point discrimination test (mm) & \\
$\quad$ Injured finger & $4.9(4-6)$ \\
$\quad$ Uninjured finger & $4.5(4-6)$ \\
Moving two-point discrimination test (mm) & $3.9(3-5)$ \\
$\quad$ Injured finger & $3.5(3-4)$ \\
Uninjured finger & $4 / 7 / 4 / 0$ \\
Satisfaction, excellent/good/fair/poor & \\
\hline
\end{tabular}

(3-5 years old, $n=10)$ [6]. The data from the patients documented by the chart review are summarized in Table 1 . The mean age was 3.0 years (range, $1-5$ years). The mean diameters of the artery and vein were $0.49 \mathrm{~mm}$ (range, $0.4-0.7 \mathrm{~mm}$ ) and $0.34 \mathrm{~mm}$ (range, $0.3-0.5 \mathrm{~mm}$ ), respectively. The mean time of the operation was 142 minutes (range, 105-240 minutes).

Table 2 shows the growth rate of the dimensions of the flap and that of the distal phalanx of the injured and contralateral uninjured digits, which were measured using data from the time of injury and the last outpatient visit, as well as the results of the two-point discrimination test of the injured and uninjured fingers at the last outpatient visit. Satisfaction (with the shape of the flap, fingertip sensation, performance in daily life, and second toe) of the surgical site was evaluated through a questionnaire provided to patients and parents.

The sensory recovery of the flap was compared with the sensory perception of a normal digit through two-point discrimination testing. No statistically significant difference was found in the growth of the distal phalanx and the sensation of the flap between the injured and contralateral uninjured fingers, as shown in Table 3.

Table 4 shows Spearman nonparametric correlation coeffi- 
cients between the growth rate of the flap dimensions and distal phalanx length. The Spearman correlation coefficient was 0.932, thus showing a strong positive relationship, and the correlation between the growth rates was statistically significant $(\mathrm{P}=$ 0.001). To sum up, the flap grew to match the growth of the distal phalanx. Lastly, Table 5 summarizes the postoperative com-

Table 3. Comparison of distal phalanx growth and sensation between injured and uninjured fingers $(n=15)$

\begin{tabular}{|lccc|}
\hline \multirow{2}{*}{ Variable } & \multicolumn{3}{c|}{ Mean (range) } \\
\cline { 2 - 3 } & $\begin{array}{c}\text { Injured } \\
\text { finger }\end{array}$ & $\begin{array}{c}\text { Uninjured } \\
\text { finger }\end{array}$ & \\
\hline Growth rate of distal phalanx (\%) & $43.2(0-93.1)$ & $47.6(0-95.4)$ & 0.616 \\
Static 2PD test (mm) & $4.9(4.0-6.0)$ & $4.5(4.0-6.0)$ & 0.261 \\
Dynamic 2PD test (mm) & $3.9(3.0-5.0)$ & $3.5(3.0-4.0)$ & 0.161 \\
\hline 2PD test, two-point discrimination test. & & \\
a)Student t-test and the Mann-Whitney U test. & & \\
\hline
\end{tabular}

Table 4. Spearman nonparametric coefficient correlation between growth rate of the flap dimension and the distal phalanx length

\begin{tabular}{|c|c|c|}
\hline \multicolumn{2}{|c|}{ Spearman nonparametric correlation } & $\begin{array}{l}\text { Growth rate of distal } \\
\text { phalanx length }\end{array}$ \\
\hline \multirow[t]{3}{*}{ Growth rate of flap dimension } & Correlation coefficient & 0.932 \\
\hline & P-value & $0.001^{\text {a) }}$ \\
\hline & Number & 15 \\
\hline
\end{tabular}

plications. There was no total loss of the flap or signs of donor site problems, such as wound dehiscence or scar contracture.

\section{Case 1}

A 2-year-old girl injured by a jujube sorting machine presented with index and middle finger amputation of the right hand, and replantation was performed as an emergency operation on the day of the injury. Partial necrosis occurred in the replanted middle finger of the right hand, resulting in a tip defect with bone exposure. To cover the defect, partial second toe pulp free flap surgery was performed from the ipsilateral second toe. The flap survived, and she was discharged without any further operations (Fig. 2A and B).

In 26 months of outpatient follow-up, the distal phalanx grew at a similar rate to that of the contralateral finger, as did the flap. According to the two-point discrimination test and a survey ad-

\section{Table 5. Postoperative complications}

\begin{tabular}{|lc|}
\hline Variable & No. (\%) \\
\hline Flap survival & $18(100)$ \\
Re-exploration (thrombosis, spasm, congestion) & $1(6)$ \\
Flap loss & $2(11)$ \\
Partial (>25\%) & $2(11)$ \\
Total (>75\%) & 0 \\
Complications & $7(39)$ \\
Sensory (cold intolerance, pain, tingling sensation) & $3(17)$ \\
Nail deformity & $4(22)$ \\
Donor site problem & 0 \\
\hline
\end{tabular}

\section{Fig. 2. Case 1}

(A) A 2-year-old girl suffered from $1.2 \times 1.1 \mathrm{~cm}$ soft tissue defect after replantation on the tip of right middle finger with tuft bone exposure. (B) Soft tissue coverage with partial second toe pulp free flap on the defect. (C, D) At the 26 months follow-up, the flap appears slightly bulky but shows adequate contour and a joint crease. (E, F) At the 26 months follow-up, the second toe showed uneasily noticeable scar.
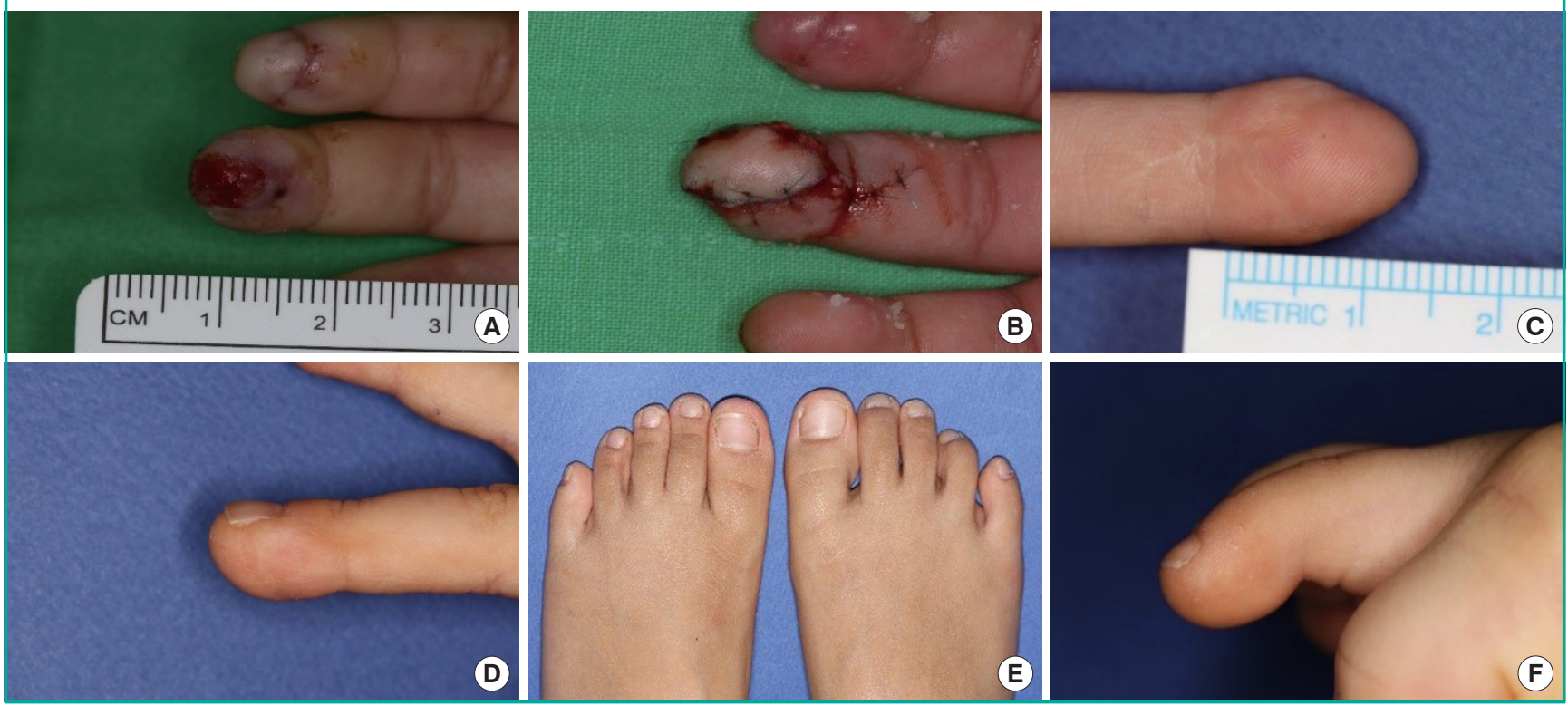


\section{Fig. 3. Case 2}

(A) A 5-year-old boy had $1.5 \times 1.2 \mathrm{~cm}$ soft defect after replantation on the tip of left index finger with bone exposure. (B) The defect was covered with a partial second toe pulp free flap. (C, D) At the 18 years follow-up, the flap and distal phalanx appears slightly short but was difficult to easily notice the differences. $(E, F)$ At the 18 years follow-up, the second toe showed scarring that was difficult to see.
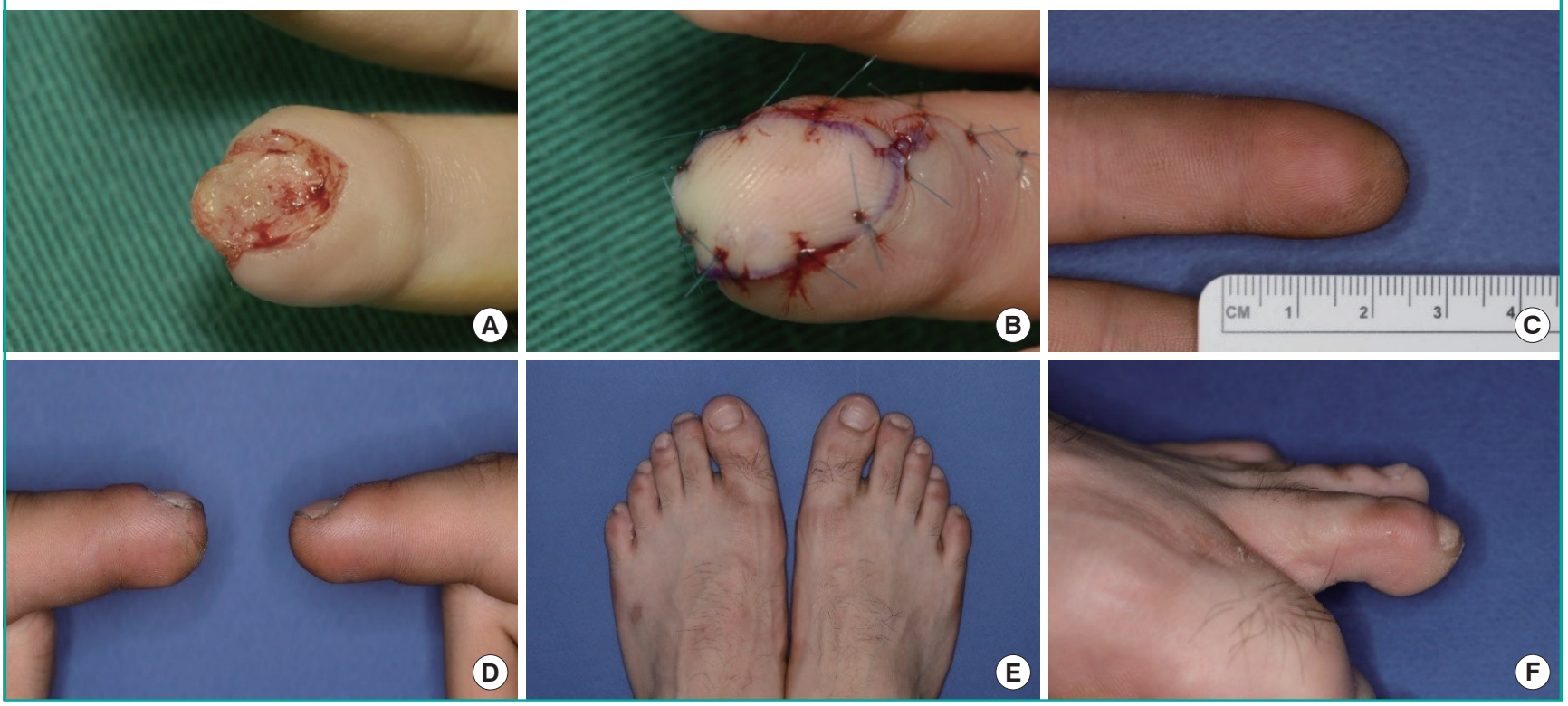

ministered to the parents and patient, sensation in the fingertip was almost normal. However, after discharge, the patient was reluctant to use her injured hand for about 3 years, although she felt no discomfort in using it in everyday life afterwards. There was no donor-site morbidity, and signs of surgery were difficult to see without careful observation (Fig. 2C-F).

\section{Case 2}

A 5-year-old boy's hand was crushed by a revolving door. His left index finger was completely amputated at the tip level and emergency replantation was performed on the day of the injury. Necrosis in the replanted finger caused a soft tissue defect with tuft bone exposure. Soft tissue coverage was achieved using a partial second toe pulp free flap from the right foot. The flap survived without any additional procedures (Fig. $3 \mathrm{~A}$ and B).

After 18 years, the distal phalanx and the flap had grown, with similar proportions to the contralateral digit. According to the two-point discrimination test and a survey of the patient, the sensation in the fingertip was almost normal, without discomfort in daily life and work. There was no donor-site morbidity, and it was difficult to notice that surgery had been performed without careful observation (Fig. 3C-F).

\section{DISCUSSION}

This study investigated the long-term outcomes of partial second toe pulp free flaps performed for fingertip defects in early childhood, and found that these flaps provide an excellent option for fingertip reconstruction in young children, as well as adults.

There are many articles regarding toe pulp free flaps, but this is the first paper to describe the feasibility and outcomes of the free flap procedure with respect to children alone. Furthermore, other studies regarding pediatric free tissue transfers included patients close to adulthood, over the age of 12 [9-11], but this study was limited to children 5 years of age or younger. All the flaps survived, the rates of complications and flap necrosis were low, and as the patients grew, the flaps and the distal phalanges also grew at a similar rate to the other digits. The patients and parents were satisfied with the sensation at the injured sites, which recovered to almost the same degree as the opposite side.

In early childhood, physical activities increase as children become more independent. A highly competitive society puts pressure on parents or caregivers, who continuously observe and protect their children, to pursue a career; this leaves their children at a greater risk of accidents resulting from inattention from their caregivers. As children's hands are small and vulnerable to injuries, if their fingers get jammed between the hinges of a closet, for example, they may suffer severe injury, as their fingers have less resistance than those of adults or older children. Furthermore, unlike school-age children, upper respiratory infections occur more frequently in this period, increasing the risks associated with the use of general anesthesia.

The options for reconstruction of the fingertip in children are 
limited if amputation does occur, bearing in mind that surgeons must consider several factors such as low compliance, the small vascular diameter, and parents' preference. If the amputated distal part is present, a replantation or composite graft should be performed, but if there is no amputated stump or in the presence of severe damage of the amputated part or defects caused by necrosis after initial surgery, other options should be considered.

Dressing, healing by secondary intention, and skin grafting are noninvasive procedures that may restore the child's fingertip, but could lead to growth retardation and deformity of the nail and fingertip, causing stress for the child during school life [12]. The volar V-Y advancement flap and island flap are somewhat limited depending on the size of the defect. The thenar flap and cross-finger flap are difficult to maintain during a long immobilization period and require secondary surgery for division of the flap $[13,14]$.

Although surgical techniques and postoperative care may be challenging due to the difficulty of pedicle isolation, microvascular free tissue transfer could be a good option for the treatment of fingertip injuries in children depending on factors related to the quality of life, the desires of the parents, and psychological effects [15].

Among the many microvascular reconstruction methods, a partial second toe pulp free flap has the most similar characteristics to the fingertip. It is glabrous, durable, and of a similar color. Furthermore, sensory recovery after surgery is outstanding [16]. Finally, this flap maintains its round shape as the flap grows, without contracture, and there is little donor-site morbidity [17].

In our institution, partial second toe pulp free flaps have been performed for 22 years, mainly in adult patients with fingertip defects, ever since the authors first introduced the procedure [8]. Furthermore, as we have advanced this surgical technique, we have been able to shorten the operation time, improve the survival rate of the flap, reduce complications, and expand the indications of partial second toe pulp free flap to children. Due the small diameter of vessels in pediatric patients, highly meticulous and skillful microsurgical manipulation was needed. However, intraoperative vascular spasm was less of a problem than in adults. Although postoperative care was harder than with adult patients, there were no postoperative complications such as vascular spasm and recipient and donor wound dehiscence.

In Table 3, we compared the growth rate of the distal phalanx between the injured and uninjured contralateral digits in patients who were traceable, and found similar or slightly less growth in the injured finger; however, the difference was not statistically significant. This finding is presumed to be due to the fact that there were mostly tiny fractures of the distal phalanx without any damage to the epiphysis, and outstanding restoration is possible because children rarely have comorbidities such as diabetes, hypertension, and smoking $[15,18]$.

A strong positive relationship between the growth of the flap and that of the distal phalanx was observed, which means that the flap had grown to match the growth of the distal phalanx. It seems that reduced wound contracture and early mobilization promoted the growth of the flap [19]. Fingerprint recognition for smartphones was also available.

The tip sensation in the injured finger was similar to that in the contralateral side according to two-point discrimination tests, both static and dynamic, conferring it an "excellent" rating according to the American Association for Hand Surgery criteria. In addition, according to the questionnaire given to the patients and parents, the patients felt comfortable in their daily lives. However, there were some cases where the patient's subjective feeling on the injured side was slightly different from that on the normal side. Cold intolerance, a tingling sensation, nail deformity, and visible scar were identified in some cases, but such instances were fewer than those reported in a previous study [9]. Furthermore, as the children grew up, the donor second toe showed a difficult-to-notice scar and the patients and parents did not complain of discomfort on the second toe.

This study has several limitations. First, due to the limited inclusion criteria, the number of cases was small; thus, the significance of our statistical analysis is limited. Second, the results may have been affected by bias because of the fact that this was a retrospective comparative study, not a randomized controlled trial. For this reason, it will be necessary to conduct prospective randomized studies with larger sample sizes in the future. Lastly, since the follow-up period was not consistent among the cases, there was large variation (3-23 years old) in patients' ages at the last observation. For toddlers (1-2 years old), it was difficult to conduct an accurate two-point discrimination test and to administer the survey satisfactorily. Therefore, it will be necessary to gauge the results in future after all the patients have grown up. In summary, the use of a partial second toe pulp free flap in children, as well as in adults, is an excellent fingertip reconstruction method that is feasible and effective with a high flap survival rate, good functional outcomes, and high patient and parent satisfaction.

\section{NOTES}

\section{Conflict of interest}

No potential conflict of interest relevant to this article was reported. 


\section{Ethical approval}

The study was approved by the Institutional Review Board of Gwangmyeong Sungae Hospital (IRB No. KIRB-2019-N-010) and performed in accordance with the principles of the Declaration of Helsinki. Written informed consents were obtained.

\section{Patient consent}

The patient's parents and patients provided written informed consent for the publication and the use of images.

\section{Author contribution}

Conceptualization: all authors. Data curation: MK Hong, DC Lee, MS Choi. Formal analysis: MK Hong, MS Choi. Methodology: all authors. Project administration: all authors. Visualization: MK Hong, DC Lee, MS Choi, SY Roh. Writing - original draft: MK Hong, DC Lee, MS Choi. Writing - review \& editing: all authors.

\section{ORCID}

Min Ki Hong

Dong Chul Lee

Min Suk Choi

Sung Hoon Koh

Jin Soo Kim

Si Young Roh

Kyung Jin Lee https://orcid.org/0000-0002-8587-7420 https://orcid.org/0000-0003-4211-6901 https://orcid.org/0000-0003-0411-7006 https://orcid.org/0000-0002-8094-2561 https://orcid.org/0000-0003-3369-2974 https://orcid.org/0000-0002-8625-6124 https://orcid.org/0000-0002-9448-8291

\section{REFERENCES}

1. Macgregor DM, Hiscox JA. Fingertip trauma in children from doors. Scott Med J 1999;44:114-5.

2. Doraiswamy NV, Baig H. Isolated finger injuries in children: incidence and aetiology. Injury 2000;31:571-3.

3. Menckhoff C, Kimpel N, Kaminstein D. Pediatric hand injuries, part II: lacerations, amputations, bites, and nail-bed injuries. Pediatr Emerg Med Rep 2009;14:121-31.

4. Gellman H. Fingertip-nail bed injuries in children: current concepts and controversies of treatment. J Craniofac Surg 2009;20:1033-5.

5. Martin C, Gonzalez del Pino J. Controversies in the treatment of fingertip amputations: conservative versus surgical reconstruction. Clin Orthop Relat Res 1998;(353):63-73.

6. Kliegman R, Stanton B, Behrman RE, et al. Nelson textbook of pediatrics. 20th ed. Philadelphia: Elsevier; 2016.

7. Jeon BJ, Yang JW, Roh SY, et al. Microsurgical reconstruction of soft-tissue defects in digits. Injury 2013;44:356-60.

8. Lee DC, Kim JS, Ki SH, et al. Partial second toe pulp free flap for fingertip reconstruction. Plast Reconstr Surg 2008; 121:899-907.

9. Canales F, Lineaweaver WC, Furnas H, et al. Microvascular tissue transfer in paediatric patients: analysis of 106 cases. $\mathrm{Br}$ J Plast Surg 1991;44:423-7.

10. Upton J, Guo L. Pediatric free tissue transfer: a 29-year experience with 433 transfers. Plast Reconstr Surg 2008;121: 1725-37.

11. Aboelatta YA, Aly HM. Free tissue transfer and replantation in pediatric patients: technical feasibility and outcome in a series of 28 patients. J Hand Microsurg 2013;5:74-80.

12. Foucher G, Boulas HJ, Braga Da Silva J. The use of flaps in the treatment of fingertip injuries. World J Surg 1991;15: 458-62.

13. McGregor IA. Flap reconstruction in hand surgery: the evolution of presently used methods. J Hand Surg Am 1979;4: $1-10$.

14. Rockwell WB, Lister GD. Soft tissue reconstruction: coverage of hand injuries. Orthop Clin North Am 1993;24:41124.

15. Akcal A, Karsidag S, Sucu DO, et al. Microsurgical reconstruction in pediatric patients: a series of 30 patients. Ulus Travma Acil Cerrahi Derg 2013;19:411-6.

16. Lin $\mathrm{CH}$, Lin YT, Sassu P, et al. Functional assessment of the reconstructed fingertips after free toe pulp transfer. Plast Reconstr Surg 2007;120:1315-21.

17. Kim HS, Lee DC, Kim JS, et al. Donor-site morbidity after partial second toe pulp free flap for fingertip reconstruction. Arch Plast Surg 2016;43:66-70.

18. Nunley JA, Spiegl PV, Goldner RD, et al. Longitudinal epiphyseal growth after replantation and transplantation in children.J Hand Surg Am 1987;12:274-9.

19. Cho JY, Suh HS, Hong JP. Do skin perforator flaps accommodate foot growth in children after reconstruction? J Reconstr Microsurg 2016;32:650-6. 